Relations industrielles

Industrial Relations

\title{
L'unité syndicale et la CSN
}

\section{Jean Marchand}

Volume 16, numéro 2, avril 1961

URI : https://id.erudit.org/iderudit/1021812ar

DOI : https://doi.org/10.7202/1021812ar

Aller au sommaire du numéro

\section{Éditeur(s)}

Département des relations industrielles de l'Université Laval

ISSN

0034-379X (imprimé)

1703-8138 (numérique)

Découvrir la revue

Citer ce document

Marchand, J. (1961). L'unité syndicale et la CSN. Relations industrielles /

Industrial Relations, 16(2), 267-269. https://doi.org/10.7202/1021812ar

Tous droits réservés ( Département des relations industrielles de l’Université Laval, 1961
Ce document est protégé par la loi sur le droit d'auteur. L’utilisation des services d'Érudit (y compris la reproduction) est assujettie à sa politique d'utilisation que vous pouvez consulter en ligne.

https://apropos.erudit.org/fr/usagers/politique-dutilisation/ 
force ouvrière totale ne dépassera pas l'âge de vingt-cinq ans. Si la situation actuelle persiste, le tiers de tous ces jeunes gens n'aura reçu qu'une instruction de niveau élémentaire (ou même moins encore) et qu'un autre tiers n'aura pas complété la douzième année. Si l'on considère qu'en février 1960, 44 pour cent de tous les chômeurs n'avaient pas terminé les classes de l'école élémentaire et que 26 pour cent d'autres chômeurs étaient allés seulement jusqu'à la huitième année, la relation vitale qui existe entre l'insuffisance d'instruction et le chômage ne peut que sauter aux yeux.

Le Conseil a recommandé que les femmes ayant des personnes à charge et les vieux travailleurs sur le point de prendre la retraite soient libres de choisir de se tenir à l'écart du marché du travail ou d'y entrer. Une aide devrait être fournie par le truchement de l'assistance publique générale ou d'autres programmes de soutien du revenu pour ceux qui jugent préférable de rester chez eux. Quant aux autres, il faudrait prévoir à leur intention des programmes de formation et d'orientation pour les aider à trouver un emploi lucratif, et soutenir certains services, si nécessaire, comme par exemple, les garderies de jour ou les services d'aides familiales pour s'occuper des enfants des mères qui travaillent.

Finalement, tout devrait être mis en oeuvre, par le truchement de programmes de réadaptation, pour permettre aux handicapés, de s'acquitter de leur obligation de subvenir à leurs propres besoins, dans les limites de leurs possibilités. Ceux qui ne peuvent soutenir la concurrence sur le marché ordinaire du travail devraient bénéficier d'un revenu complémentaire suffisant et de services communautaires, tels que les ateliers de travail protégés.

Selon le Conseil, la preuve indiscutable est faite que bon nombre des personnes qui se trouvent actuellement dans ces groupes spéciaux et constituent de ce fait une lourde charge pour la société, pourraient moyennant une aide, arriver à se suffire ou tout au moins à mener une existence utile et à apporter une contribution digne d'intérêt à l'économie nationale.

\section{L'UNITÉ SYNDICALE ET LA CSN}

\section{Jean Marchand, président général de la CSN}

Le congrès de 1955, de notre Confédération, s'est prononcé en faveur du principe de l'unité syndicale au Canada. Ultérieurement, il a mandaté un comité spécial pour entamer des pourparlers avec le Congrès du travail du Canada afm d'atteindre cet objectif. Plusieurs rencontres eurent lieu entre les représentants ds deux groupes mais, à date, rien ne nous permet d'espérer qu'une solution rapide sera trouvée aux difficultés rencontrées au cours des négociations.

UNITÉ SYNDICALE, RÊVE OU RÉALITÉ

Une vue panoramique de la situation syndicale dans le monde occidental, nous révèle qu'il y a très peu de pays où l'unité syndicale a été réalisée. Pourquoi? 
Le syndicalisme ne poursuivant pas exclusivement des objectifs économiques, (salaires, vacances, conditions de travail, etc.) mais se proposant de transformer toute la société, il s'en suit que tout le monde ne conçoit pas de la même façon la reconstruction sociale, économique et politique. Ainsi, les communistes n'ont pas la même conception que les chrétiens ou les socialistes. Il se crée donc des organisations qui s'inspirent d'idéologies différentes.

Cette diversité n'est pas un mal puisqu'elle correspond à une réalité et qu'elle est la conséquence de l'activité libre de l'homme. Elle est même la source d'un dynamisme qu'on ne rencontre pas dans les pays où l'on impose une pensée officielle unique.

Tout le problème réside à concilier la liberté d'option entre plusieurs idéologies et l'unité des cadres.

Les Américains ont cru contourner la difficulté en faisant du syndicalisme nonidéologique. L'expérience démontre que ce n'est pas là chose facile. On n'étouffe pas la vie par une décision de congrès. Les syndicats américains présentent une diversité qui loin de s'atténuer s'en va en s'accentuant. Personne n'oserait comparer l'Union des ouvriers de l'automobile (U.A.W.) avec l'Union des charpentiers-unis d'Amérique. Les différences ne s'expliquent pas exclusivement par la nature des industries. Nous sommes en présence de deux unions qui ont des conceptions différentes de l'organisation économique des Etats-Unis, du syndicalisme et de la vie politique. Voilà sans doute pourquoi les syndicats américains ne parviennent pas à réaliser l'unité organique sur la base de l'industrie. La seule unité que la fusion a apporté est l'unité des corps dit «législatifs 》 qui, comme on le sait, sont les organismes les plus faibles dans les structures du syndicalisme nord-américain.

La situation canadienne ne diffère pas de la situation américaine. C'est dans notre confédération que nous retrouvons la plus grande unité.

L'unité syndicale n'est pas un rêve mais c'est un équilibre extrêmement difficile à atteindre et qui ne doit pas se réaliser au détriment de la liberté syndicale sinon, on risque de vider le syndicalisme de son sens en le réduisant à un rôle de simple agence de négociations collectives.

\section{LES DÉLATs}

On considère parfois que les négociations entre le CTC et la CSN prennent beaucoup de temps. En effet, elles se poursuivent depuis plus de cinq ans. Mais, les pourparlers entre le C.I.O. et l'A.F. of L. n'ont-ils pas duré quinze ans! Ainsi en fut-il des tractations d'unité entre le Congrès des métiers et du travail et le Congrès canadien du travail. Il n'y a donc pas encore lieu de s'alarmer ou de se désespérer.

\section{CONDITION FONDAMENTALE}

Lorsque le congrès de notre Confédération s'est prononcé en faveur de l'unité syndicale, il a posé une condition formelle: la CSN ne doit pas disparaitre et il ne 
peut être question de fusion. Autrement dit, le congrès était prêt à réaliser l'unité mais en sauvegardant les cadres de notre mouvement et son intégrité doctrinale. Cest pourquoi nous avons demandé et obtenu que le CTC accorde à la CSN le statut d'une \& union nationale $>$ au sens de la constitution du CTC. Ce statut nous offrait les garanties exigées par notre congrès. Malheureusement, par suite de difficultés constitutionnelles, le CTC ne semble plus vouloir nous affilier sous ce statut. Nous devrons dons chercher une nouvelle formule qui tiendra compte des exigences de nos propres congrès.

\section{Conclusion}

Il est souhaitable que nous réalisions l'unité syndicale au Canada mais pas à n'importe quel prix. Cette unité ne doit pas se bâtir sur un mouvement dévitalisé, standardisé et qui étouffe les tendances idéologiques qui sont le ferment même de la pensée syndicale. Les structures syndicales ne pourront jamais être copiées sur les structures de la grande entreprise capitaliste car, dans le premier cas, ce sont des hommes et non des intérêts qui sont en jeu. L'unité dans la diversité, telle est la formule, mais comment $y$ parvenir?

\section{RECENSIONS - BOOK REVIEWS}

Human Relations and Modern Management. Ed. by E. M. Hugh-Jones. Amsterdam: North-Holland, Publishing Co., P.O. Box 103, 1958. 256 pp. $\$ 5.30$.

Ce petit volume est le premier d'une série en préparation chez le même éditeur sur I'Economique industrielle. Dans une perspective de relations humaines, il tâche d'établir le dialogue entre l'homme d'affaires et l'adepte des sciences de l'homme. Il possède une saveur internationale toute spéciale, car y ont contribué, en des essais originaux, quatre économistes ou sociologues anglais, trois américains et un hollandais. Au delà des divergences prévues, le volume trouve son unité dans l'étude d'un ordre de problèmes: ceux qui relèvent de la direction d'hommes au travail.

Dans un premier article (\& Human Relations and Fundamental Principles of Management »), J. L. Meij présente, à l'intérieur et au coeur même d'une théorie de la direction, une esquisse des relations humaines. Il établit succinctement un rapport mathématique entre les dimensions d'une entreprise et le nombre de (managers s à divers niveaux qui sont nécessaires à cette entre- prise; un tel rapport pourra varier grâce à la spécialisation de certaines fonctions de direction.

L'article de W. H. Scott sur \& The Factory as a Social System 》 est bien dans la ligne des Mayoïtes, et notamment de Warner et de Gardner, au niveau du petit groupe de travail. Toutefois, l'auteur se hâte d'affirmer que sto regard employees simply as members of small face-to-face groups is to ignore the importance of their other affiliations and particularly those, such as trade union membership, which derive from the fact that a work force is divided into a number of occupational categories \$. Scott décrit plus loin diverses catégories de la structure des occupations, et discute la dichtomie structure formelle-structure naturelle.

L'essai de Robert L. Kahn touche aux relations humaines au niveau de latelier. Il rend compte, à ce plan, de la recherche effectuée au Survey Research Center de l'Université du Michigan par lui-même et ses collègues, notamment Daniel Katz et Rensis Likert. Il s'agit de concilier la rationalité presque machinale du fonctionnement de l'entreprise et les besoins humains variés de ceux qui y oeuvrent 